01

\title{
Высокочастотная асимптотика спектрального распределения энергии равновесного излучения в вырожденной электронной плазме
}

\author{
(C) В.Б. Бобров \\ Объединенный институт высоких температур РАН, \\ 125412 Москва, Россия \\ Национальный исследовательский университет „МЭИ“, \\ 111250 Москва, Россия \\ e-mail: vic5907@mail.ru
}

(Поступило в Редакцию 1 июня 2017 г.)

Показано, что в вырожденной электронной плазме асимптотическое поведение спектрального распределения энергии равновесного излучения в области высоких частот принципиально отличается от формулы Планка в силу степенного характера убывания по частоте. При этом определяющим является учет собственного магнитного момента электронов.

DOI: 10.21883/JTF.2018.02.45402.2370

\section{Введение}

Формула Планка [1], определяющая спектральное распределение энергии равновесного излучения, соответствует идеализированной модели абсолютно черного тела, представляющего собой свободную от вещества полость, заполненную излучением и ограниченную абсолютно поглощающим веществом. При этом не рассматриваются эффекты взаимодействия фотонов с ограничивающим полость веществом, учет которых необходим для установления состояния термодинамического равновесия [2]. Тем самым распределение Планка описывает объемные характеристики равновесного излучения абсолютно черного тела в пренебрежении поверхностными эффектами, обусловленными наличием вещества.

Аналогичная ситуация фактически имеет место и при рассмотрении макроскопического тела, находящегося в тепловом равновесии с окружающим его „черным“ излучением [2]. В решении этой задачи, имеющей непосредственное отношение к закону Кирхгофа, достигнуты большие успехи (см. подробнее [3-5] и цитированную там литературу). При этом поглощательная способность тела, являясь поверхностной характеристикой, рассматривается, по сути, как объемная.

С другой стороны, исследованию спектрального распределения энергии излучения в самом веществе, находящемся в состоянии термодинамического равновесия с излучением, практически не уделялось внимания (см. [6-9] и цитированную там литературу). Это обусловлено, как принято считать, необходимостью решения вопроса об энергии электромагнитного поля в поглощающей среде [10]. С формальной точки зрения проблема заключается в установлении соотношения между энергией электромагнитного поля и общим выражением для тепловых потерь в среде в нестационарных условиях, когда средние значения напряженностей электромагнитного поля отличны от нуля и изменяются во времени. Это приводит в конечном итоге к рассмотрению только областей прозрачности [10-13]. При таком рассмотрении решение задачи возможно для равновесного состояния среды в статическом электромагнитном поле или, в крайнем случае, при слабой зависимости электромагнитного поля от времени [10-13].

Однако случай равновесной материальной среды, представляющей собой совокупность электромагнитного поля и вещества (заряженных частиц) является в этом смысле исключением. В такой системе тепловые потери отсутствуют, т.е. поглощение электромагнитного поля уравновешено его испусканием. При этом, средние значения напряженностей электромагнитного поля равны нулю [14]. Это позволяет решить вопрос о спектральном распределении энергии равновесного излучения в материальной среде $[8,9]$.

\section{Спектральное распределение энергии равновесного излучения в материальной среде}

Рассмотрим материальную среду, представляющую собой совокупность заряженных частиц и фотонов. Термодинамические свойства такой материальной среды, занимающей объем $V$, полностью определяются ее термодинамическим потенциалом Гиббса [15]

$$
\Omega\left(T, V,\left\{\gamma_{a}\right\}\right)=-T \ln Z\left(T, V,\left\{\gamma_{a}\right\}\right),
$$

где $T$ - температура рассматриваемой системы в энергетических единицах, $\gamma_{a}-$ химический потенциал для заряженных частиц сорта $a$, которые характеризуются массой $m_{a}$, зарядом $z_{a} e$, спином $s_{a}$ и собственным магнитным моментом $\mu_{a}$. Здесь и далее для простоты рассматривается нерелятивистская система заряженных частиц $[2,15]$. Химический потенциал для фотонов, как обычно [2], считается равным нулю. 
По определению большая статистическая сумма $Z\left(T, V,\left\{\gamma_{a}\right\}\right)$ равна

$$
Z\left(T, V,\left\{\gamma_{a}\right\}\right)=T_{r \exp }\left\{-\left(\hat{H}-\sum_{a} \gamma_{a} \hat{N}_{a}\right) / T\right\},
$$

где $\hat{H}$ - гамильтониан системы в представлении вторичного квантования:

$$
\hat{H}=\hat{H}_{\mathrm{part}}+\hat{H}_{\mathrm{ph}}
$$

$H_{\text {part }}$ - гамильтониан взаимодействующих заряженных частиц в квантованном электромагнитном поле

$$
\begin{aligned}
\hat{H}_{\mathrm{part}}= & \sum_{a} \frac{\hbar^{2}}{2 m_{a}} \int d^{3} r\left(\nabla+\frac{i z_{a} e}{\hbar} \hat{\mathbf{A}}(\mathbf{r})\right) \\
& \times \hat{\Psi}_{a}^{+}(\mathbf{r})\left(\nabla-\frac{i z a e}{\hbar} \hat{\mathbf{A}}(\mathbf{r})\right) \hat{\Psi}_{a}(\mathbf{r}) \\
& -\sum_{a} \int d^{3} r \hat{\Psi}_{a}^{+}(\mathbf{r}) \hat{\boldsymbol{\mu}}_{a} \hat{\Psi}_{a}(\mathbf{r}) \operatorname{rot} \hat{\mathbf{A}}(\mathbf{r})+\hat{H}_{\mathrm{Coul}}
\end{aligned}
$$

$H_{\text {Coul }}$ - гамильтониан кулоновского взаимодействия заряженных частиц

$$
\begin{aligned}
\hat{H}_{\text {Coul }}= & \frac{1}{2} \sum_{a, b} \int d^{3} r_{1} d^{3} r_{2} u_{a b}\left(\left|\mathbf{r}_{1}-\mathbf{r}_{2}\right|\right) \\
& \times \hat{\Psi}_{a}^{+}\left(\mathbf{r}_{1}\right) \hat{\Psi}_{b}^{+}\left(\mathbf{r}_{2}\right) \hat{\Psi}_{b}\left(\mathbf{r}_{2}\right) \hat{\Psi}_{a}\left(\mathbf{r}_{1}\right)
\end{aligned}
$$

$u_{a b}(r)=\left(z_{a} z_{b} e^{2}\right) / r-$ потенциал кулоновского взаимодействия заряженных частиц сортов $a$ и $b, \boldsymbol{\mu}_{a}-$ оператор собственного магнитного момента для частиц сорта $a, \hat{\Psi}_{a}^{ \pm}(\mathbf{r})$ и $\hat{\Psi}_{a}(\mathbf{r})$ - соответственно полевые операторы рождения и уничтожения для заряженных частиц сорта $a, \hat{N}_{a}=\int d^{3} r \hat{\Psi}_{a}^{+}(\mathbf{r}) \hat{\Psi}_{a}(\mathbf{r})-$ оператор полного числа частиц сорта $a, \hat{\mathbf{A}}(\mathbf{r})$ - оператор векторного потенциала, соответствующий квантованному электромагнитному полю

$$
\begin{aligned}
\hat{\mathbf{A}}(\mathbf{r})= & c \sum_{\mathbf{k}, \lambda}\left(\frac{2 \pi \hbar}{\omega_{\mathbf{k}} V}\right)^{1 / 2}\left\{\mathbf{e}_{\mathbf{k}}^{(\lambda)} \hat{c}_{\mathbf{k}, \lambda} \exp (i \mathbf{k r})\right. \\
& \left.+\mathbf{e}_{\mathbf{k}}^{(\lambda) *} \hat{c}_{\mathbf{k}, \lambda}^{+} \exp (-i \mathbf{k r})\right\},
\end{aligned}
$$

$c$ - скорость света, $\mathbf{e}_{\mathbf{k}}^{(\lambda)}-$ векторы поляризации фотонов, которые удовлетворяют условиям

$$
\mathbf{e}_{\mathbf{k}}^{(\lambda)} \mathbf{k}=0, \quad \sum_{\lambda=1}^{2} e_{\mathbf{k} \alpha}^{(\lambda)} e_{\mathbf{k} \beta}^{(\lambda) *}=\delta_{\alpha, \beta}-\frac{k_{\alpha} k_{\beta}}{k^{2}}
$$

Гамильтониан свободного поля излучения $\hat{H}_{\mathrm{ph}}$ определяется равенством

$$
\hat{H}_{\mathrm{ph}}=\sum_{\mathbf{k}, \lambda} \hbar \omega_{\mathbf{k}} \hat{c}_{\mathbf{k}, \lambda}^{+} \hat{c}_{\mathbf{k}, \lambda}, \quad \omega_{\mathbf{k}}=c|\mathbf{k}|
$$

где операторы рождения $\hat{c}_{\mathbf{k}, \lambda}^{+}$и уничтожения $\hat{c}_{\mathbf{k}, \lambda}$ для фотонов с импульсом $\hbar \mathbf{k}$ и поляризацией $\lambda=1,2$ удовлетворяют перестановочным соотношениям

$$
\left[\hat{c}_{\mathbf{k}, \lambda}, \hat{c}_{\mathbf{k}^{\prime}, \lambda^{\prime}}^{+}\right]=\delta_{\mathbf{k}, \mathbf{k}^{\prime}} \delta_{\lambda, \lambda^{\prime}} .
$$

В рамках статистической квантовой электродинамики вычисление термодинамического потенциала Гиббса (1) основано на использовании функциональных методов теории возмущений по взаимодействию заряженных частиц между собой и с квантованным электромагнитным полем, так что величина $\Omega\left(T, V,\left\{\gamma_{a}\right\}\right)$ является функционалом от функций Грина для частиц и фотонов. При этом равновесная фотонная функция Грина однозначно определяется диэлектрической проницаемостью среды $\varepsilon^{\operatorname{tr}}(k, \omega)[16]$.

В соответствии с (1)-(8) средняя энергия для рассматриваемой системы с гамильтонианом (3) равна

$$
E=\langle\hat{H}\rangle=\left\langle\hat{H}_{\text {part }}\right\rangle+\left\langle\hat{H}_{\mathrm{ph}}\right\rangle,
$$

где угловые скобки обозначают усреднение с большим каноническим распределением Гиббса [15-17].

Тем самым величину $E_{\mathrm{ph}} \equiv\left\langle\hat{H}_{\mathrm{ph}}\right\rangle$ можно рассматривать как среднюю энергию равновесного излучения в материальной среде в отличие от средней энергии взаимодействующих заряженных частиц в квантованном электромагнитном поле, определяемой как $E_{\text {part }} \equiv\left\langle\hat{H}_{\text {part }}\right\rangle$.

Как и при рассмотрении идеального газа фотонов, описываемого формулой Планка, средняя энергия равновесного излучения в веществе может быть представлена в виде

$$
E_{\mathrm{ph}}=V \sum_{\lambda} \int \frac{d^{3} k}{(2 \pi)^{3}} \hbar \omega_{\mathbf{k}} f(\mathbf{k}, \lambda)=V \int_{0}^{\infty} \varepsilon_{\omega}\left(T,\left\{\gamma_{a}\right\}\right) d \omega
$$

где $f(\mathbf{k}, \lambda) \equiv\left\langle\hat{c}_{\mathbf{k}, \lambda}^{+} \hat{c}_{\mathbf{k}, \lambda}\right\rangle$ - точная равновесная функция распределения фотонов по импульсам $\hbar \mathbf{k}$ в материальной среде [8]. При этом спектральное распределение энергии излучения в веществе $\varepsilon_{\omega}\left(T,\left\{\gamma_{a}\right\}\right)$ зависит не только от частоты флуктуаций электромагнитного поля $\omega$ и температуры среды $T$, как это имеет место в формуле Планка для идеального газа фотонов, но и от характеристик вещества, а именно набора химических потенциалов заряженных частиц $\left\{\gamma_{a}\right\}$.

Используя методы квантовой теории поля [16] для рассматриваемой системы, можно показать $[8,9]$, что для спектрального распределения энергии излучения $\varepsilon_{\omega}\left(T,\left\{\gamma_{a}\right\}\right)$ в материальной среде справедливо соотношение

$$
\varepsilon_{\omega}\left(T,\left\{\gamma_{a}\right\}\right)=\varepsilon_{\omega}^{(0)}(T)+\Delta \varepsilon_{\omega}\left(T,\left\{\gamma_{a}\right\}\right)
$$

где величина $\varepsilon_{\omega}^{(0)}(T)$ определяется формулой Планка

$$
\varepsilon_{\omega}^{(0)}(T)=\frac{\hbar}{\pi^{2} c^{3}} \frac{\omega^{3}}{\exp (\hbar \omega / T)-1},
$$


а функция $\Delta \varepsilon_{\omega}\left(T,\left\{\gamma_{a}\right\}\right)-$ равенством

$$
\begin{aligned}
\Delta \varepsilon_{\omega}\left(T,\left\{\gamma_{a}\right\}\right) & =\frac{\hbar \omega^{3}}{\pi^{2} c^{3}} \operatorname{cth}\left(\frac{\hbar \omega}{2 T}\right) \\
& \times\left(\frac{c^{5}}{\pi \omega} \int_{0}^{\infty} d k k^{4} \frac{\operatorname{Im} \varepsilon^{\operatorname{tr}}(k, \omega)}{\left|\varepsilon^{\operatorname{tr}}(k, \omega) \omega^{2}-c^{2} k^{2}\right|^{2}}-\frac{1}{2}\right) .
\end{aligned}
$$

Таким образом, в спектральном распределении энергии излучения в материальной среде имеется вклад $\Delta \varepsilon_{\omega}\left(T,\left\{\gamma_{a}\right\}\right)$ (13), обусловленный наличием вещества (заряженных частиц). Этот вклад полностью определяется поперечной диэлектрической проницаемостью $\varepsilon^{\operatorname{tr}}(k, \omega)$ рассматриваемой системы. При этом соотношение (13) справедливо только для однородной и изотропной системы, линейные электромагнитные свойства которой однозначно определяются продольной $\varepsilon^{l}(k, \omega)$ и поперечной $\varepsilon^{\operatorname{tr}}(k, \omega)$ диэлектрическими проницаемостями [18].

Другими словами, функция $\varepsilon^{\operatorname{tr}}(k, \omega)$ определяет не только оптические свойства материальной среды [18], но и объемные равновесные характеристики излучения в ней (см., например, $[19,20])$.

\section{Поперечная диэлектрическая проницаемость вырожденной электронной плазмы}

Общее выражение для функции $\varepsilon^{\operatorname{tr}}(k, \omega)$ для рассматриваемой системы имеет вид $[16,21]$

$$
\begin{gathered}
\varepsilon^{\operatorname{tr}}(k, \omega)=1-\frac{\omega_{p}^{2}}{\omega^{2}}-\frac{4 \pi}{\omega^{2}} \Pi^{\operatorname{tr}}(k, \omega), \\
\Pi^{\operatorname{tr}}(k, \omega)=\frac{1}{2}\left(\delta_{\alpha \beta}-\frac{k_{\alpha} k_{\beta}}{k^{2}}\right) \Pi_{\alpha \beta}(\mathbf{k}, \omega),
\end{gathered}
$$

где $\omega_{p}=\left(\sum_{a} 4 \pi z_{a}^{2} e^{2} n_{a} / m_{a}\right)^{1 / 2}-$ плазменная частота, $n_{a}$ - плотность числа частиц сорта $a$. Тензорный поляризационный оператор $\Pi_{\alpha \beta}(\mathbf{k}, \omega)$ представляет собой неприводимую по одной „линии“ (как кулоновского взаимодействия заряженных частиц, так и фотонной функции Грина) часть запаздывающей тензорной функция Грина „ток-ток“ $\Phi_{\alpha \beta}^{R}(\mathbf{k}, \omega)$, которая равна

$$
\begin{gathered}
\Phi_{\alpha \beta}^{R}(\mathbf{k}, \omega)=\int d^{3} r \exp (-i \mathbf{k r}) \int_{0}^{\infty} d t \exp (i \omega t) \Phi_{\alpha \beta}^{R}(\mathbf{r}, t), \\
\Phi_{\alpha \beta}^{R}\left(\mathbf{r}_{1}-\mathbf{r}_{2}, t\right)=-\frac{i}{\hbar}\left\langle\left[\hat{J}_{\alpha}\left(\mathbf{r}_{1}, t\right), \hat{J}_{\beta}\left(\mathbf{r}_{2}, 0\right)\right]\right\rangle .
\end{gathered}
$$

Здесь $\hat{\mathbf{J}}(\mathbf{r}, t)$ - векторный оператор плотности тока заряженных частиц в представлении Гейзенберга,

$$
\hat{\mathbf{J}}(\mathbf{r})=\hat{\mathbf{J}}^{(d)}(\mathbf{r})+\hat{\mathbf{J}}^{(p)}(\mathbf{r})-\sum_{a} \frac{z_{a}^{2} e^{2}}{m_{a} c} \hat{\mathbf{A}}(\mathbf{r}) \hat{\Psi}_{a}^{+}(\mathbf{r}) \hat{\Psi}_{a}(\mathbf{r}),
$$

$$
\hat{\mathbf{J}}^{(d)}(\mathbf{r})=\sum_{a} \frac{i \hbar z_{a} e}{2 m_{a}}\left\{\left(\nabla \hat{\Psi}_{a}^{+}(\mathbf{r})\right) \hat{\Psi}_{a}(\mathbf{r})-\hat{\Psi}_{a}^{+}(\mathbf{r})\left(\nabla \hat{\Psi}_{a}(\mathbf{r})\right)\right\}
$$

$$
\hat{\mathbf{J}}^{(p)}(\mathbf{r})=\operatorname{rot} \sum_{a} \hat{\Psi}_{a}^{+}(\mathbf{r}) \hat{\boldsymbol{\mu}}_{a} \hat{\Psi}_{a}(\mathbf{r}) .
$$

Как следует из соотношений (14)-(19) в общем случае вычисление поперечной диэлектрической проницаемости $\varepsilon^{\operatorname{tr}}(k, \omega)$ рассматриваемой системы является весьма сложной задачей. Это в еще большей степени относится к вычислению интеграла в (13) при определении величины $\Delta \varepsilon_{\omega}\left(T,\left\{\gamma_{a}\right\}\right)$.

Чтобы упростить рассмотрение, учтем, что постоянная тонкой структуры $\alpha=e^{2} / \hbar c \simeq 1 / 137$, которая характеризует силу взаимодействия между заряженными частицами и фотонами, является малым параметром. Это обстоятельство является основой для использования в квантовой статистической электродинамике теории возмущений (диаграммной техники), связанной с представлением средних значений физических величин в виде функциональных рядов по степеням $\alpha$ [16].

Тем самым, согласно существующим представлениям при вычислении поперечной диэлектрической проницаемости $\varepsilon^{\operatorname{tr}}(k, \omega)$ в широкой области термодинамических параметров, можно ограничиться рассмотрением „нулевого“ приближения по параметру $\alpha$, т.е. рассматривать функцию $\varepsilon^{\operatorname{tr}}(k, \omega)(14)-(19)$ для системы заряженных частиц, пренебрегая их взаимодействием с фотонами.

В этом случае тензорный поляризационный оператор $\Pi_{\alpha \beta}(k, \omega)$ можно представить в виде

$$
\Pi_{\alpha \beta}(\mathbf{k}, \omega)=\Pi_{\alpha \beta}^{(d d)}(\mathbf{k}, \omega)+\Pi_{\alpha \beta}^{(p p)}(\mathbf{k}, \omega),
$$

где величина $\Pi_{\alpha \beta}^{(d d)}(\mathbf{k}, \omega)$ отвечает диамагнитной части, a величина $\Pi_{\alpha \beta}^{(p p)}(\mathbf{k}, \omega)$ - парамагнитной части поляризационного оператора $\Pi_{\alpha \beta}(\mathbf{k}, \omega)$. Тензорные функции $\Pi_{\alpha \beta}^{(d d)}(\mathbf{k}, \omega)$ и $\Pi_{\alpha \beta}^{(p p)}(\mathbf{k}, \omega)$ определяются аналогично соотношениям (16), (17) с точностью до замены оператора $\hat{\mathbf{J}}(\mathbf{r})$ (18) на операторы $\hat{\mathbf{J}}^{(d)}(\mathbf{r})(19)$ и $\hat{\mathbf{J}}^{(p)}(\mathbf{r})$ (20) соответственно. При этом обе функции $\Pi_{\alpha \beta}^{(d d)}(\mathbf{k}, \omega)$ и $\Pi_{\alpha \beta}^{(p p)}(\mathbf{k}, \omega)$ являются неприводимыми по одной линии кулоновского взаимодействия заряженных частиц в „,k-канале“. Кроме того, функция $\Pi_{\alpha \beta}^{(p p)}(\mathbf{k}, \omega)$ также является неприводимой по двум линиям кулоновского взаимодействия [21].

Это означает, что приближение идеального газа при вычислении тензорных функций $\Pi_{\alpha \beta}^{(d d)}(\mathbf{k}, \omega)$ и $\Pi_{\alpha \beta}^{(p p)}(\mathbf{k}, \omega)$ соответствует системе заряженных частиц, которая характеризуется интегральной малостью кулоновского взаимодействия:

$$
z_{a}^{2} e^{2} /\left\langle r_{a}\right\rangle\left\langle k_{a}\right\rangle \ll 1
$$

где $\left\langle r_{a}\right\rangle=\left(4 \pi n_{a} / 3\right)^{-1 / 3}$ - среднее расстояние между частицами, $\left\langle k_{a}\right\rangle$ - средняя кинетическая энергия, приходящаяся на одну частицу сорта $a$ [18]. К таким системам относятся как газовая полностью ионизованная плазма [22], так и электронная плазма в жидких металлах [23]. 
Согласно (15)-(21), в приближении идеального газа (индекс $(0))$ величину $\Pi_{0}^{\mathrm{tr}}(k, \omega)(15)$ можно представить в виде [21]

$$
\begin{gathered}
\Pi_{0}^{\mathrm{tr}}(k, \omega)=\sum_{a}\left\{\Phi_{a}^{(d d)}(k, \omega)+\Phi_{a}^{(p p)}(k, \omega)\right\}, \\
\Phi_{a}^{(d d)}(k, \omega)=\frac{1}{2}\left(2 s_{a}+1\right) \frac{z_{a}^{2} e^{2} \hbar^{2}}{m_{a}^{2}} \int \frac{d^{3} p}{(2 \pi)^{3}}\left(p^{2}-\frac{(\mathbf{p} \cdot \mathbf{k})^{2}}{k^{2}}\right) \\
\times \frac{f_{a}(\mathbf{p}-\mathbf{k} / 2)-f_{a}(\mathbf{p}+\mathbf{k} / 2)}{\hbar \omega+\epsilon_{a}(\mathbf{p}-\mathbf{k} / 2)-\epsilon_{a}(\mathbf{p}+\mathbf{k} / 2)+i 0}, \\
\Phi_{a}^{(p p)}(k, \omega)=k^{2} \frac{\left(2 s_{a}+1\right) s_{a}\left(s_{a}+1\right)}{3}\left(\frac{\mu_{a} c}{s_{a}}\right)^{2} \int \frac{d^{3} p}{(2 \pi)^{3}} \\
\times \frac{f_{a}(\mathbf{p}-\mathbf{k} / 2)-f_{a}(\mathbf{p}+\mathbf{k} / 2)}{\hbar \omega+\epsilon_{a}(\mathbf{p}-\mathbf{k} / 2)-\epsilon_{a}(\mathbf{p}+\mathbf{k} / 2)+i 0} .
\end{gathered}
$$

Здесь $\epsilon_{a}(p)=\hbar^{2} p^{2} / 2 m_{a}-$ энергия частицы, $f_{a}(p)-$ функция распределения по импульсам для частиц сорта $a$, которая определяется распределениями Ферми-Дирака или Бозе-Эйнштейна в зависимости от спина частицы $s_{a}$. Величина химического потенциала $\gamma_{a}$ при заданной температуре связана с плотностью числа частиц $n_{a}$ равенством

$$
n_{a}=\left(2 s_{a}+1\right) \int \frac{d^{3} p}{(2 \pi)^{3}} f_{a}(p),
$$

так что выполняется условие квазинейтральности

$$
\sum_{a} z_{a} e n_{a}=0
$$

При рассмотрении жидких металлов условие (21) может быть принято только для электронной подсистемы, так как взаимодействие между ионами в жидких металлах является сильным. Однако благодаря большой разнице в массах электронов и ионов для описания электромагнитных свойств жидких металлов можно ограничиться рассмотрением только электронной плазмы, которая понимается как система электронов в компенсирующем положительном фоне ионов (см. (26)) [23].

Кроме того, электронная плазма в жидких металлах считается полностью вырожденной с силу условия

$$
T \ll \epsilon_{\mathrm{F}},
$$

где $\epsilon_{F}=\hbar^{2} k_{\mathrm{F}}^{2} / 2 m_{e}-$ энергия Ферми, $k_{\mathrm{F}}=\left(3 \pi^{2} n_{e}\right)^{1 / 3}-$ волновой вектор Ферми для системы электронов.

По этой причине при рассмотрении различных свойств жидких металлов в подавляющем большинстве случаев для описания электронной плазмы используется формальный предел $T \rightarrow 0(T=+0)$, понимаемый в смысле справедливости условия (27) [23].

В рамках приближения $T=+0$ функция распределения Ферми-Дирака для электронов имеет вид

$$
f\left(p<k_{\mathrm{F}}\right)=1, f\left(p=k_{\mathrm{F}}\right)=1 / 2, f\left(p>k_{\mathrm{F}}\right)=0,
$$

что позволяет получить явные аналитические выражения для функций $\Phi_{e}^{(d d)}(k, \omega)(23)$ и $\Phi_{e}^{(p p)}(k, \omega)(24)$ (см. подробнее [24]):

$$
\begin{aligned}
& \operatorname{Re} \Phi_{e}^{(d d)}(k, \omega)=\frac{3 \omega_{e}^{2}}{32 \pi}\left\{3\left(\frac{\omega}{k \nu_{\mathrm{F}}}\right)^{2}+\left(\frac{k}{2 k_{\mathrm{F}}}\right)^{2}-\frac{5}{3}\right. \\
& +\frac{k_{\mathrm{F}}}{2 k}\left[1-\left(\frac{k}{k_{\mathrm{F}}}\right)^{2}\left(\Delta^{(-)}(k, \omega)\right)^{2}\right]^{2} \ln \left[\frac{k_{\mathrm{F}}+k \Delta^{(-)}(k, \omega)}{k_{\mathrm{F}}-k \Delta^{(-)}(k, \omega)}\right] \\
& -\frac{k_{\mathrm{F}}}{2 k}\left[1-\left(\frac{k}{k_{\mathrm{F}}}\right)^{2}\left(\Delta^{(+)}(k, \omega)\right)^{2}\right]^{2} \\
& \left.\times \ln \left[\frac{k_{\mathrm{F}}+k \Delta^{(+)}(k, \omega)}{k_{\mathrm{F}}-k \Delta^{(+)}(k, \omega)}\right]\right\}, \\
& \operatorname{Im} \Phi_{e}^{(d d)}(k, \omega)=0, \quad\left|\Delta^{(-)}\right| \geq k_{\mathrm{F}} / k ; \\
& =-\frac{3 \omega_{e}^{2}}{64}\left(\frac{k_{\mathrm{F}}}{k}\right)\left[1-\left(\frac{k}{k_{\mathrm{F}}}\right)^{2}\left(\Delta^{(-)}\right)^{2}\right]^{2}, \\
& \left|\Delta^{(-)}\right| \leq k_{\mathrm{F}} / k \leq \Delta^{(+)}, \\
& -\frac{3 \omega_{e}^{2}}{16}\left(\frac{\omega}{k v_{\mathrm{F}}}\right)\left[1-\left(\frac{\omega}{k v_{\mathrm{F}}}\right)^{2}-\left(\frac{k}{2 k_{\mathrm{F}}}\right)^{2}\right]^{2}, \Delta^{(+)} \leq k_{\mathrm{F}} / k, \\
& \operatorname{Re} \Phi_{e}^{(p p)}(k, \omega)=-\frac{3 \omega_{e}^{2}}{32 \pi}\left(\frac{k}{k_{\mathrm{F}}}\right)^{2}\left\{1-\frac{k_{\mathrm{F}}}{2 k}\left[1-\left(\frac{k}{k_{\mathrm{F}}}\right)^{2}\right.\right. \\
& \times\left(\Delta^{(-)}(k, \omega)\right)^{2} \ln \left[\frac{k_{\mathrm{F}}+k \Delta^{(-)}(k, \omega)}{k_{\mathrm{F}}-k \Delta^{(-)}(k, \omega)}\right] \\
& \left.+\frac{k_{\mathrm{F}}}{2 k}\left[1-\left(\frac{k}{k_{\mathrm{F}}}\right)^{2}\left(\Delta^{(+)}(k, \omega)\right)^{2}\right] \ln \left[\frac{k_{\mathrm{F}}+k \Delta^{(+)}(k, \omega)}{k_{\mathrm{F}}-k \Delta^{(+)}(k, \omega)}\right]\right\}, \\
& \operatorname{Im} \Phi_{e}^{(p p)}(k, \omega)=0, \quad\left|\Delta^{(-)}\right| \geq k_{\mathrm{F}} / k \\
& =-\frac{3 \omega_{e}^{2}}{64}\left(\frac{k_{\mathrm{F}}}{k}\right)\left[1-\left(\frac{k}{k_{\mathrm{F}}}\right)^{2}\left(\Delta^{(-)}\right)^{2}\right] \text {, } \\
& \left|\Delta^{(-)}\right| \leq k_{\mathrm{F}} / k \leq \Delta^{(+)}, \\
& -\frac{3 \omega_{p}^{2}}{64}\left(\frac{k}{k_{\mathrm{F}}}\right)\left(\frac{\hbar \omega}{\varepsilon_{\mathrm{F}}}\right), \quad \Delta^{(+)} \leq k_{\mathrm{F}} / k .
\end{aligned}
$$

Здесь $v_{\mathrm{F}}=\hbar k_{\mathrm{F}} / m_{e}-$ скорость Ферми, $\omega_{e}=$ $=\left(4 \pi n_{e} e^{2} / m_{e}\right)^{1 / 2}-$ электронная плазменная частота,

$$
\Delta^{(-/+)}(k, \omega)=\left(\frac{m_{e} \omega}{\hbar k^{2}}\right) \mp\left(\frac{1}{2}\right) .
$$

При выводе (29)-(33) учтено, что собственный магнитный момент электрона, который характеризуется спином $s_{e}=1 / 2$, равен $\mu_{e}=-\mu_{\mathrm{B}}$, где $\mu_{\mathrm{B}}=|e| \hbar / 2 m_{e} c-$ магнетон Бора.

При этом следует иметь в виду, что даже при выполнении условия (27) поперечная диэлектрическая проницаемость вырожденной электронной плазмы $\varepsilon^{\operatorname{tr}}(k, \omega)$ 
зависит также от двух независимых безразмерных параметров $\beta_{k}$ и $\beta_{\omega}$ :

$$
\beta_{k}=\epsilon_{k} / T=\left(k \Lambda_{e}\right)^{2} / 4 \pi, \quad \beta_{\omega}=\hbar \omega / T,
$$

где $\Lambda_{e}=\left(2 \pi \hbar^{2} / m_{e} T\right)^{1 / 2}$ - тепловая длина волны де Бройля для электронов. Это означает, что приближение $T=+0$ не может быть использовано для описания функции $\varepsilon^{\mathrm{tr}}(k, \omega)$ в области малых волновых векторов $\beta_{k} \ll 1$ и низких частот $\beta_{\omega} \ll 1$. Другими словами, соотношения (29)-(33) справедливы, когда

$$
\beta_{k} \gg 1, \quad \beta_{\omega} \gg 1 \text {. }
$$

Это означает, что необходимо оценить возможность использования соотношений (29)-(33) для вычисления спектрального распределения энергии равновесного излучения $\varepsilon_{\omega}(11)-(13)$ в вырожденной электронной плазме. Непосредственно из (22)-(24) нетрудно убедиться, что вклад области малых волновых векторов $\beta_{k} \ll 1$ в величину $\Delta \varepsilon_{\omega}(13)$ чрезвычайно мал при условии

$$
\hbar \omega / \epsilon_{\mathrm{F}} \gg 1 .
$$

С учетом (27) условие (36) удовлетворяет второму неравенству в (35).

Таким образом, представленные выше результаты для поперечной диэлектрической проницаемости могут быть использованы для анализа асимптотического поведения спектрального распределения энергии равновесного излучения в вырожденной электронной плазме в области высоких частот.

\section{Высокочастотная асимптотика спектрального распределения энергии равновесного излучения в вырожденной электронной плазме}

Прежде всего отметим, что в области высоких частот (36) с учетом (27), (35) вклад величины $\varepsilon_{\omega}^{(0)}(T)$, определяемой формулой Планка (12), в спектральное распределение энергии $\varepsilon_{\omega}(11)$ экспоненциально мал.

Кроме того, при рассмотрении области высоких частот (36), понимаемых для вырожденной электронной плазмы как предел $\omega \rightarrow \infty$, соотношение (13) для функции $\Delta \varepsilon_{\omega}$ можно существенно упростить. Дело в том, что, согласно (14), (22)-(24), (29)-(33), при фиксированном значении волнового вектора $k$ справедливы равенства

$$
\lim _{\omega \rightarrow \infty} \operatorname{Re} \varepsilon^{\operatorname{tr}}(k, \omega)=1, \quad \lim _{\omega \rightarrow \infty} \operatorname{Im} \varepsilon^{\operatorname{tr}}(k, \omega)=0 .
$$

Далее рассмотрим функцию

$$
F(\omega)=\frac{c^{5}}{\pi \omega^{5}} \int_{0}^{\infty} d k k^{4} \frac{\operatorname{Im} \varepsilon^{\operatorname{tr}}(k, \omega)}{\left|\varepsilon^{\operatorname{tr}}(k, \omega)-c^{2} k^{2} / \omega^{2}\right|^{2}} .
$$

В пределе $\omega \rightarrow \infty$ эта функция имеет вид

$$
\begin{gathered}
\left.F(\omega)\right|_{\omega \rightarrow \infty}=F(\infty)+\Delta F(\omega), \quad F(\infty)=\lim _{\omega \rightarrow \infty} F(\omega), \\
\lim _{\omega \rightarrow \infty} \Delta F(\omega)=0 .
\end{gathered}
$$

Чтобы вычислить величину $F(\infty)(40)$, необходимо учесть, что, согласно (37)

$$
\begin{gathered}
\left.\left(\frac{\operatorname{Im} \varepsilon^{\operatorname{tr}}(k, \omega)}{\left(\operatorname{Re} \varepsilon^{\operatorname{tr}}(k, \omega)-c^{2} k^{2} / \omega^{2}\right)^{2}+\left(\operatorname{Im} \varepsilon^{\operatorname{tr}}(k, \omega)\right)^{2}}\right)\right|_{\omega \rightarrow \infty} \\
\rightarrow \pi \delta\left(1-c^{2} k^{2} / \omega^{2}\right),
\end{gathered}
$$

где $\delta(x)$ - дельта-функция Дирака. Следовательно,

$$
F(\infty)=\lim _{\omega \rightarrow \infty} F(\omega)=1 / 2 .
$$

В свою очередь, для функции $\Delta F(\omega)$ (39), согласно (37), в пределе $\omega \rightarrow \infty$ можно записать

$$
\left.\Delta F(\omega)\right|_{\omega \rightarrow \infty}=\left.\frac{c^{3}}{\pi \omega^{5}} \int_{0}^{\infty} d k k^{4} \operatorname{Im} \varepsilon^{\operatorname{tr}}(k, \omega)\right|_{\omega \rightarrow \infty} .
$$

Тогда, подставляя (38)-(42) в (13), находим

$$
\left.\left.\Delta \varepsilon_{\omega}\left(T,\left\{\gamma_{a}\right\}\right)\right|_{\omega \rightarrow \infty} \rightarrow \frac{\hbar c^{2}}{\pi^{3} \omega^{2}} \int_{0}^{\infty} d k k^{4} \operatorname{Im} \varepsilon^{\operatorname{tr}}(k, \omega)\right|_{\omega \rightarrow \infty}
$$

Таким образом, появляется возможность установить степень влияния вырожденной электронной плазмы на спектральное распределение энергии равновесного излучения в области высоких частот, для которой с учетом (14), (20), (27), (30), (32) имеем

$$
\begin{gathered}
\left.\Delta \varepsilon_{\omega}\left(\epsilon_{\mathrm{F}}\right)\right|_{\omega \rightarrow \infty} \rightarrow \Delta \varepsilon_{\omega}^{(d d)}+\Delta \varepsilon_{\omega}^{(p p)}, \\
\Delta \varepsilon_{\omega}^{(d d)}=\left.\frac{3 \hbar c^{2} \omega_{e}^{2} k_{\mathrm{F}}^{5}}{16 \pi^{2} \omega^{4}} Y^{(d d)}(W)\right|_{W \rightarrow \infty}, \\
\Delta \varepsilon_{\omega}^{(p p)}=\left.\frac{3 \hbar c^{2} \omega_{e}^{2} k_{\mathrm{F}}^{5}}{16 \pi^{2} \omega^{4}} Y^{(p p)}(W)\right|_{W \rightarrow \infty}, \\
Y^{(d d)}(W)=\int_{\sqrt{W+1}-1}^{\sqrt{W+1}+1} d x x^{3}\left[1-\frac{x^{2}}{4}\left(\frac{W}{x^{2}}-1\right)^{2}\right]^{2}, \\
Y^{(p p)}(W)=\int_{\sqrt{W+1}+1}^{\sqrt{W+1}-1} d x x^{5}\left[1-\frac{x^{2}}{4}\left(\frac{W}{x^{2}}-1\right)^{2}\right]^{2},
\end{gathered}
$$

где $W=\hbar \omega / \epsilon_{\mathrm{F}}$. Чтобы упростить вычисление асимптотического поведения функций $Y^{(d d)}(W)$ и $Y^{(p p)}(W)$ в пределе $W \rightarrow \infty$, произведем в интегралах (47) и (48) сначала замену переменных $x=y \sqrt{W+1}$, а затем $z=(y-1)$. В результате получаем

$$
Y^{(d d)}(W)=(W+1)^{2} \int_{-(W+1)^{-1 / 2}}^{(W+1)^{-1 / 2}} d x \psi^{(d d)}(z, W),
$$




$$
\begin{aligned}
\psi^{(d d)}(z, W)= & (z+1)^{3}\left[1-(W+1) \frac{(z+1)^{2}}{4}\right. \\
& \left.\times\left(\frac{W}{(W+1)(z+1)^{2}}-1\right)^{2}\right]^{2}, \\
Y^{(p p)}(W)= & (W+1)^{3} \int_{-(W+1)^{-1 / 2}}^{(W+1)^{-1 / 2}} d z \psi^{(p p)}(z, W), \\
\psi^{(p p)}(z, W)= & (z+1)^{5}\left[1-(W+1) \frac{(z+1)^{2}}{4}\right. \\
& \left.\times\left(\frac{W}{(W+1)(z+1)^{2}}-1\right)^{2}\right],
\end{aligned}
$$

Следовательно,

$$
\begin{aligned}
\left.Y^{(d d)}(W)\right|_{W \rightarrow \infty} & =\left.\left(\frac{2(W+1)^{2}}{\sqrt{W+1}} \lim _{Z \rightarrow 0} \psi^{(d d)}(z, W)\right)\right|_{W \rightarrow \infty} \\
& \rightarrow 2 W^{3 / 2} \\
\left.Y^{(p p)}(W)\right|_{W \rightarrow \infty} & =\left.\left(\frac{2(W+1)^{3}}{\sqrt{W+1}} \lim _{Z \rightarrow 0} \psi^{(p p)}(z, W)\right)\right|_{W \rightarrow \infty} \\
& \rightarrow 2 W^{5 / 2} .
\end{aligned}
$$

Подставляя (51), (52) соответственно в (45), (46), находим

$$
\begin{aligned}
& \Delta \varepsilon_{\omega}^{(d d)}=\frac{6 m_{e} c^{2} \omega_{e}^{2}}{\pi^{2} v_{\mathrm{F}}^{3}}\left(\frac{\epsilon_{\mathrm{F}}}{\hbar \omega}\right)^{5 / 2}, \\
& \Delta \varepsilon_{\omega}^{(p p)}=\frac{6 m_{e} c^{2} \omega_{e}^{2}}{\pi^{2} v_{\mathrm{F}}^{3}}\left(\frac{\epsilon_{\mathrm{F}}}{\hbar \omega}\right)^{3 / 2} .
\end{aligned}
$$

Таким образом, в области высоких частот асимптотическое поведение спектрального распределения энергии равновесного излучения $\left.\Delta \varepsilon_{\omega}\left(\epsilon_{\mathrm{F}}\right)\right|_{\omega \rightarrow \infty}(44)$ в вырожденной электронной плазме полностью определяется наличием вещества (электронов), причем наиболее существенным является вклад „парамагнитной“ функции $\Delta \varepsilon_{\omega}^{(p p)}(53)$ :

$$
\left.\Delta \varepsilon_{\omega}\left(\epsilon_{\mathrm{F}}\right)\right|_{\omega \rightarrow \infty} \rightarrow \Delta \varepsilon_{\omega}^{(p p)} .
$$

Это означает, что для корректного описания характеристик равновесного излучения в материальной среде необходимо последовательно учитывать собственный магнитный момент частиц вещества. Аналогичные результаты имеют место и при рассмотрении газовой полностью ионизованной плазмы (см. подробнее $[9,25]$ ).

В заключение отметим, что в применении к жидким металлам полученные выше результаты имеют место только в отношении так называемых „коллективизированных“ электронов. Для описания электронных состояний, локализованных около ядер, требуется учет эффектов сильного взаимодействия, что выходит за рамки данной работы.

Автор благодарен А.А. Рухадзе и С.А. Тригеру за полезные обсуждения. Настоящая работа выполнена при финансовой поддержке РФФИ (грант № 17-02-00573).

\section{Список литературы}

[1] Planck M. // Ann. der Phys. 1901. Vol. 309. N 3. P. 553-563.

[2] Ландау Л.Д., Лифшич Е.М. Статистическая физика. Часть 1. М.: Наука, 1976. 584 с.

[3] Левин М.Л., Рытов С.М. Теория равновесных тепловых флуктуаций в электродинамике. М.: Наука, 1967. 308 с.

[4] Волокитин А.И., Перссон Б.Н.Дж. // УФН. 2007. Т. 177. Вып. 9. С. 921-951.

[5] Виноградов Е.А., Дорофеев И.А. // УФН. 2009. Т. 179. Вып. 5. C. 449-485.

[6] Bobrov V.B. // J. Phys.: Cond. Matt. 1990. Vol. 2. N 31. P. 6695-6698.

[7] Бобров В.Б. // ТМФ. 1991. Т. 88. Вып. 1. С. 141-145.

[8] Бобров В.Б., Соколов И.М., Тригер С.А. // Письма в ЖЭТФ. 2015. Т. 101. Вып. 5. С. 326-329.

[9] Бобров В.Б., Тригер С.А. // ТМФ. 2016. Т. 187. Вып. 1. C. $104-113$.

[10] Ландау Л.Д., Лифшии, Е.М. Электродинамика сплошных сред. М.: Наука, 1982. 524 с.

[11] Trigger S.A. // Phys. Lett. A. 2007. Vol. 370. N 5-6. P. 365369.

[12] Khomkin A.L., Shumikhin A.S., Trigger S.A. // J. Phys.: Conf. Ser. 2015. Vol. 653. P. 012024 (1-5).

[13] Агранович В.М., Гинзбург В.Л. Электродинамика с учетом пространственной дисперсии и теория экситонов. М: Наука, 1964. 376 с.

[14] Бараш Ю.С., Гинзбург В.Л. // УФН. 1975. Т. 116. Вып. 1. C. 5-40.

[15] Ахиезер А.И., Пелетминский С.В. Методы статистической физики. М.: Наука, 1977. 368 с.

[16] Фрадкин Е.С. // Труды ФИАН. 1965. Т. 29. С. 7-138.

[17] Бобров В.Б., Тригер С.А., Петров О.Ф. // ТВТ. 2017. Т. 55. Вып. 1. С. 154-157.

[18] Силин В.П., Рухадзе А.А. Электромагнитные свойства плазмы и плазмоподобных сред. М.: Либроком, 2013. $248 \mathrm{c}$.

[19] Агранат М.Б., Аиитков С.И., Овчинников А.В., Ситников Д.С., Юркевич А.А., Чефонов О.В., Перельман Л.Т., Анисимов С.И., Фортов В.Е. // Письма в ЖЭТФ. 2015. Т. 101. Вып. 9. С. 671-676.

[20] Дьячков Л.Г. // ТВТ. 2016. Т. 54. Вып. 1. С. 7-12.

[21] Bobrov V.B. // Physica A. 1992. Vol. 187. N 3-4. P. 603-624.

[22] Кребт В.-Д., Кремп Д., Эбелинг В., Репке Г. Квантовая статистика систем заряженных частиц. М.: Мир, 1988. $408 \mathrm{c}$.

[23] Бобров В.Б. // ТВТ. 2016. Т. 54. Вып. 3. С. 475-478.

[24] Бобров В.Б. // ТВТ. 2017. Т. 55. Вып. 4. С. 489-492.

[25] Бобров В.Б., Тригер С.А. // ТМФ. 2017. Т. 192. Вып. 3. C. $523-535$. 